\title{
The baboon syndrome after cinchocaine ointment
}

\section{Case report I}

A 48-year-old man presented with progressive, intense perianal erythematous area after an initial 3-day period of topical cream ointment application, composed of cinchocaine and policresulen, in order to treat haemorrhoids. He complained of a progressive reddish area comprising from the middle sacral third to the greater trochanter of the femur, bilaterally, to the inner part of the proximal thighs. The patient denied any systemic symptom, just local discomfort and pruritus. He used a towel to retain the significant amount of transudation formed, turning his chair wet. There was no infectious signs. Digital rectal examination was uneventful and the anal canal had no alterations. Patient confirmed not applying the ointment into the anal canal.

\section{Case report 2}

After two weeks, a 49-year-old male patient complained of perianal discomfort associated with a 7-day progressive local hyperemia after topical use of cinchocaine and policresulen ointment in order to treat a thrombosed external haemorrhoid. He denied fever or other systemic symptoms, just reporting pruritus and local intense transudation, demanding the use of several feminine napkins. The perianal hyperemic area comprised from distal sacrum to the isquiadic tuberosities and to the scrotum. There were some cutaneous erosions related to itching. The thrombosed external haemorrhoid was in a normal healing status, presenting mucosal apical disruption. There were not any infectious signs. Anal canal examination was uneventful.

Laboratory exams from both patients were normal. In the emergency room, they were administered intravenously dipirone,
Volume 10 Issue 5 - 2020

\author{
Ilario Froehner Junior \\ Colorectal Surgeon at University of Sao Paulo, Nossa Senhora \\ das Graças Hospital, Brazil
}

\begin{abstract}
Correspondence: Ilario Froehner Junior, Colorectal Surgeon at University of Sao Paulo, chairman of the Gastrointestinal Motility Laboratory at Nossa Senhora das Graças Hospital, Brazil,Tel +55 4l 996845645

Email froehnrjr@yahoo.com.br, ilario.froehner@usp.br
\end{abstract}

Received: September 04, 2020 | Published: September 15, 2020

cetoprofene, hydrocortisone and intramuscular prometazine, with moderate symptoms relief.

Suspension of cinchocaine and policresulen topic ointment was advised, associated with prednisone and fexofenadine oral intake and topic nistatin plus zinc oxide ointment, 3 times a day or after evacuation.

Both patients returned to an office evaluation after a week. A significant relief in symptoms and in the hyperemia was noted. Transudation formation has remitted on the second day and a thin cutaneous desquamation took place.

After fifteen days from the initial evaluation, hyperemic area was mild and desquamation had remitted. Both patients reported some self-limited, pruriginous small papules, scattered on their bodies.

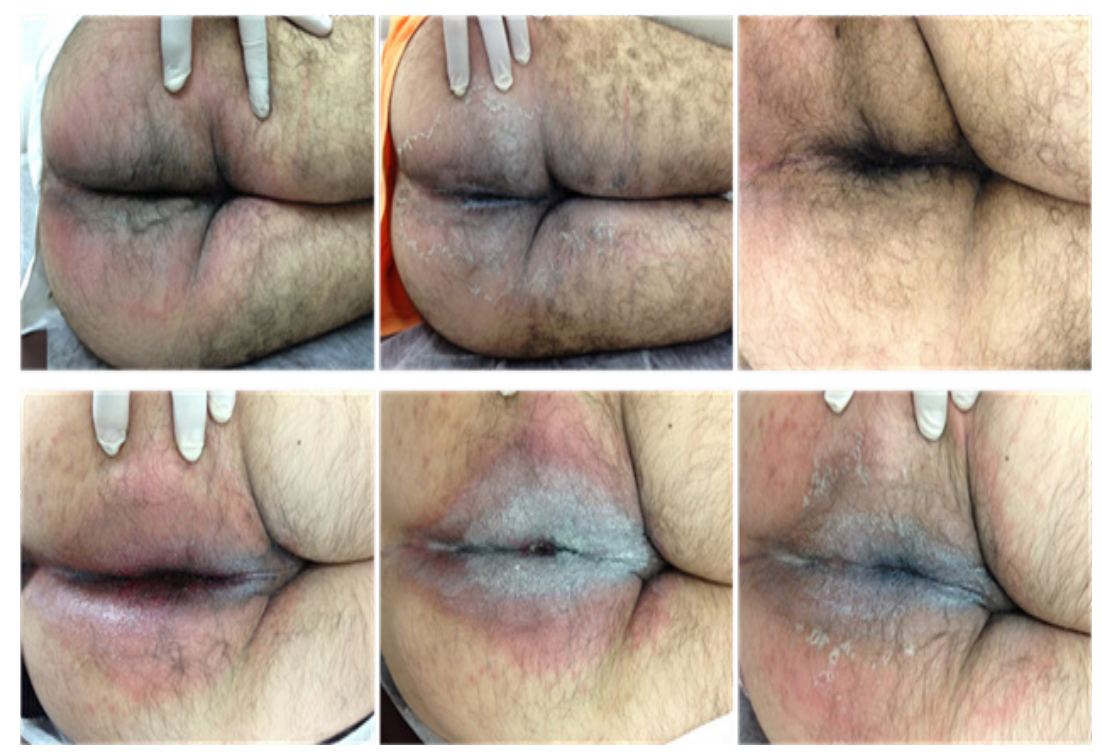

Figure I Upper images (case report I). In the left, first physical examination. The erythematous area was associated with intense transudation and comprised from the middle sacral third to the greater trochanter of the femur and to the inner portion of the thigh, bilaterally. In the centre, clinical status after a week of treatment. Symptoms were less intense and a thin desquamation took place. In the right, after I5 days of treatment, patient was asymptomatic. Lower images are related do case report 2. In the left, first physical examination. The erythematous area had also intense transudation and comprised from distal sacrum to the isquiadic tuberosities and to the scrotum. In the centre, clinical status after a week of treatment. In the right, after 15 days of treatment, symptoms were milder and a thin desquamation had started. 


\section{Discussion}

Baboon syndrome term was first introduced in 1984 to describe a mercury-induced diffuse bright red erythema resembling the red bottom of baboons. It is an allergic contact dermatitis, generally caused by mercury, nickel and balsam of Peru, typically affecting the gluteus, thighs and perineum, and may be present on other regions. ${ }^{1}$ Allergic responses to policresulen and cinchocaine are very rare, usually presenting as mild discomfort, pruritus or perianal hyperaemia. $^{2}$ Cinchocaine is a topic anesthetic from amide group, similar to lidocaine. ${ }^{3}$ Baboon syndrome after its topical application has, so far, very few cases reported. ${ }^{4}$ Cross-reaction sensitivy to lidocaine is uncommon, and it is not a contraindication to its use. ${ }^{1,5}$

\section{Conclusions}

Cinchocaine allergic responses are very rare, nevertheless, the more one substance is used, more reactions are possible to occur. Clinical correlation and early suspicion allow an accurate diagnosis and treatment. These allergic reactions do not contraindicate other amide group anesthetics use, like lidocaine.

\section{Acknowledgments}

None.

\section{Funding}

None.

\section{Conflicts of interest}

The authors have no conflicts of interest to declare.

\section{References}

1. Lee AY. Allergic contact dermatitis from dibucaine in Proctosedyl $\mathbb{}$ ointment without cross-sensitivity. Contact Dermatitis. 1998;39(5):261.

2. Mahave IG, Lobera T, Blasco A, et al. Allergic contact dermatitis caused by cinchocaine. Contact Dermatitis. 2008;58(1):55-58.

3. Froehner Junior I, Kotze PG, Rocha JG, et al. Postoperative topical analgesia of hemorrhoidectomy with policresulen and cinchocaine: a prospective and controlled study. Rev Col Bras Cir. 2014;41(2):92-98.

4. Erdmann SM, Sachs B, Merk HF. Systemic contact dermatitis from cinchocaine. Contact Dermatitis. 2001;44(4):260-261.

5. Urrutia I, Jáuregui I, Gamboa P, et al. Photocontact dermatitis from cinchocaine (dibucaine). Contact Dermatitis. 1998;39(3):139-140. 